\title{
RD-projective module whose subprojectivity domain is minimal
}

\author{
Yılmaz Durğun (D) \\ Cukurova University, Department of Mathematics, Adana, Turkey
}

\begin{abstract}
A p-indigent module is one that is subprojective only to projective modules. An RDprojective module is subprojective to any torsionfree (and flat) module. An RD-projective module $T$ is called rdp-indigent if it is subprojective only to torsionfree modules. In this work, we consider the structure of SRDP rings whose (simple) RD-projective right $R$ modules are rdp-indigent or torsionfree. Moreover, new characterizations of P-coherent rings and torsionfree rings are presented by subprojectivity domains.
\end{abstract}

Mathematics Subject Classification (2020). 16D10, 16D40, 18G25

Keywords. RD-projective module, subprojectivity domain, rdp-indigent module, torsionfree module, flat module

\section{Introduction}

In module theory, the classes of all projective and all injective modules are important subclasses of $M o d-R$ since they shed light on the whole of Mod $-R$. Accordingly, the injectivity and the projectivity of modules have been widely studied in the literature. For instance, there are some recent studies carried out to determine the injectivity (or projectivity) of an $R$-module, through a fixed module, see ([1,2,4-6,19,20,23,30]).

P-indigent modules were introduced in [20] as a dual notion of indigent modules defined in [5], and their study was furthered in [13]. Namely, while projective modules have the largest possible subprojectivity domain (all of Mod-R), the p-indigent modules are those having a domain of subprojectivity as small as possible. In this work, we focus on the subprojectivity domain of RD-projective modules.

A submodule $X_{1}$ of an $R$-module $R_{R} X$ is called relatively divisible, or briefly, an RDsubmodule if $r X_{1}=X_{1} \cap r X$ for each $r \in R$. A sequence $0 \rightarrow A_{1} \rightarrow A_{2} \rightarrow A_{3} \rightarrow 0$ of left modules is said to be RD-exact if for each $a \in R$, the sequence $\operatorname{Hom}_{R}\left(\frac{R}{R a}, A_{2}\right) \rightarrow$ $\operatorname{Hom}_{R}\left(\frac{R}{R a}, A_{3}\right) \rightarrow 0$ is exact, or equivalently $0 \rightarrow\left(\frac{R}{a R}\right) \otimes A_{1} \rightarrow\left(\frac{R}{a R}\right) \otimes A_{2}$ is exact. A module ${ }_{R} N$ is RD-injective (resp., RD-projective) if it is injective (resp., projective) according to every $\mathrm{RD}$-exact sequence $([10,17,24,31])$. According to [22], a module ${ }_{R} X$ is divisible if $\operatorname{Ext}_{R}^{1}\left(\frac{R}{R a}, X\right)=0$ for each $a \in R$. A module $N_{R}$ is called torsionfree if $\operatorname{Tor}_{1}\left(N_{R}, \frac{R}{R a}\right)=0$ for each $a \in R$. A module ${ }_{R} M$ is divisible (resp., torsionfree) if and only if each exact sequence starting (resp., ending) with ${ }_{R} M$ is an RD-exact ([24]). It is clear that $X_{R}$ is torsionfree if and only if $X^{+}$is divisible by the standard adjoint

Email address: ydurgun@cu.edu.tr

Received: 04.08.2020; Accepted: 14.09.2021 
isomorphism. For more details about divisible and torsionfree modules, we refer the reader to $[3,11,18,24,25,27]$. For commutative domains, divisible and torsionfree modules are identical with the traditional ones. Obviously, every pure exact sequence is RD-exact and so every RD-projective (RD-injective) is pure-projective (pure-injective). Recall that an $R$-module ${ }_{R} M$ is called fp-injective if $\operatorname{Ext}_{R}^{1}(N, M)=0$ for every finitely presented module ${ }_{R} N$. Moreover, every flat and fp-injective module is respectively torsionfree and divisible.

In this paper, we address some questions raised on the RD-projective modules. For an RD-projective module, the minimal subprojectivity domain is the class of torsionfree modules. Following [20], we say that an RD-projective module is relatively divisible projective indigent (shortly, rdp-indigent) if it is such a module. Unlike p-indigent modules, such an RD-projective module exists over arbitrary rings. Subprojectivity domains of the $\mathrm{RD}$-projective modules are investigated, and $\mathrm{P}$-coherent rings and torsionfree rings are characterized through subprojectivity domains of RD-projective modules. We consider the structure of rings with the property that every (singular simple) RD-projective module is rdp-indigent. Moreover, the structure of rings whose non-projective RD-projective modules are subprojective only to flat modules is investigated.

In what follows, we write ${ }_{R} \mathcal{T} \mathcal{F}$ (resp., ${ }_{R} \mathcal{F},{ }_{R} \mathcal{N},{ }_{R} \mathcal{P}$ ) for the class of all torsionfree (resp., flat, nonsingular, projective) left modules. For a module $X, E(X)$ denotes the injective envelope of $X$. The character module $\operatorname{Hom}_{\mathbb{Z}}(X, \mathbb{Q} / \mathbb{Z})$ of $X$ is denoted by $X^{+}$. For additional terminology, concepts, and results which are not mentioned here, we refer the reader to $[16,22,28]$.

\section{Subprojectivity domain of an RD-projective module}

This section is devoted to prove the basic properties about subprojectivity domains of modules that will be needed later in the paper. We start by recalling what is understood by the notion of subprojectivity. Given modules $X_{1}$ and $X_{2}, X_{1}$ is $X_{2}$-subprojective if for each epimorphism $\alpha: P \rightarrow X_{2}$ and each morphism $h: X_{1} \rightarrow X_{2}$, there exists a morphism $f: X_{1} \rightarrow P$ with $\alpha f=h$. The conditions for a module $X_{1}$ to be $X_{2^{-}}$ subprojective are given in [15,20]. For any $X \in R-M o d$, we denote by $\mathfrak{P r}^{-1}(X)$ the class $\{L \in R-M o d: \mathrm{X}$ is L-subprojective $\}$.

By [24, Proposition 2.4], a module $X$ is torsionfree if $R / R a$ is $X$-subprojective for each $a \in R$. If $X$ is torsionfree module and $Y$ is RD-projective module, then $Y$ is $X$ subprojective. For RD-projective module $M$, it is a natural question to ask how small $\mathfrak{P r}^{-1}(M)$. The next proposition shows that the domain of subprojectivity of any RDprojective module must contain at least the torsionfree modules. The following fact can be easily verified, thus its proof is omitted.

Proposition 2.1. $\bigcap_{Z \in \Lambda} \underline{\mathfrak{P r}}^{-1}(Z)=\mathcal{T F}$, where $\Lambda$ be family of all RD-projective right $R$-modules.

For an RD-projective right $R$-module $M$ :

$$
\mathcal{P}_{R} \subseteq \mathcal{F}_{R} \subseteq \mathcal{T F}_{R} \subseteq \underline{\mathfrak{P r}}^{-1}(M) .
$$

While a right perfect ring is necessary and sufficient condition to be $\mathcal{P}_{R}=\mathcal{F}_{R}$, the complete structure of a ring over which $\mathcal{P}_{R}=\mathcal{T F}_{R}\left(\right.$ or $\mathcal{T F}_{R}=\mathcal{F}_{R}$ ) is not known. However, it is clear that $R$ is a right perfect if $\mathcal{P}_{R}=\mathcal{T F}_{R}$. In terms of p-indigence, we have two new concepts for the RD-projective modules. Therefore, it makes sense to wonder about the $\mathrm{RD}$-projective modules which are subprojective relative to only torsionfree (or flat) modules.

Definition 2.2. An RD-projective right $R$-module $M$ will be called (strongly )relatively divisible projectively indigent (or an (s)rdp-indigent) in case $\mathfrak{P r}^{-1}(M)=\mathcal{T F}_{R}\left(\mathcal{F}_{R}\right)$. 
Rdp-indigent module exists over any ring. A cyclic module $X_{R} \cong R / I$ is called cyclically presented (c.p.) if $I=a R$ for some $a \in R$. Set $\mathfrak{C P}:=\oplus_{C_{i} \in \Gamma} C_{i}$, where $\Gamma$ stands for the set of all representatives of c.p. right $R$-modules. It is clear that $\mathfrak{C P}$ is an $\mathrm{RD}$-projective right $R$-module. The next result follows by definition of a torsionfree module.

Proposition 2.3. $\mathfrak{C P}$ is an rdp-indigent.

A ring $R$ is left $P$-coherent, if all principal left ideals of $R$ are finitely presented (f.p.) $([25])$.

Proposition 2.4. For each RD-projective module ${ }_{R} K, \mathfrak{P r}^{-1}(K)$ is closed under direct product if and only if $R$ is a right $P$-coherent.

Proof. $(\Leftarrow)$ We easily prove the necessity by modifying slightly the proof of [20, Proposition 2.14]. Let ${ }_{R} K$ be an RD-projective module and $\left\{A_{i}\right\}_{i \in \Phi}$ a subclass of $\mathfrak{P r}^{-1}(K)$. Let $\gamma=\left(\gamma_{i}\right)_{i \in \Phi}: K \rightarrow \prod_{i \in \Phi} A_{i}$. For each $i \in \Phi$, there exists $g_{i}: P_{i} \rightarrow \overline{A_{i}}$ with $P_{i}$ free. Since $\left\{A_{i}\right\}_{i \in \Phi}$ is a subclass of $\mathfrak{P r}^{-1}(K)$ for each $i \in \Phi$, there exists $h_{i}: K \rightarrow P_{i}$ such that $\gamma_{i}=g_{i} h_{i}$. Let $h=\left(h_{i}\right)_{i \in \Phi}: K \rightarrow \prod_{i \in \Phi} P_{i}$, and $g: \prod_{i \in \Phi} P_{i} \rightarrow \prod_{i \in \Phi} A_{i}$ be defined by $g\left(\left(x_{i}\right)_{i \in \Phi}\right)=\left(g_{i}\left(x_{i}\right)\right)_{i \in \Phi}$. It is clear that $g$ is an epimorphism and $g h=\gamma$. By [25, Theorem 2.7], $\prod_{i \in \Phi} P_{i}$ is torsionfree, hence $K$ is $\prod_{i \in \Phi} A_{i}$-subrojective by [15, Lemma 1]. $(\Rightarrow)$ For sufficiency, in particular, consider $\mathfrak{P r}^{-1}(\mathfrak{C P})$. Since $\mathfrak{C P}$ is an rdp-indigent, $R$ is a right $\mathrm{P}$-coherent by our hypothesis and [25, Theorem 2.7].

A ring $R$ is torsionfree if all (or finitely generated (f.g.)) left ideals of $R$ are torsionfree. The property of a ring being torsionfree is right-left symmetric (see [3, Corollary 3.3]). Torsionfree rings have been studied by Hattori [18] under the name left PF rings: all the principal left ideals are flat. It has been shown that $R$ is torsionfree if and only if submodules of torsionfree left modules are again torsionfree ([18, Proposition 7$])$.

Proposition 2.5. A ring $R$ is torsionfree if and only if $\mathfrak{P r}^{-1}(M)$ is closed under submodules for every $R D$-projective $R$-module $M$.

Proof. $(\Leftarrow)$ In particular, consider $\mathfrak{P r}^{-1}(\mathfrak{C P})$. $\mathfrak{C P}$ is an rdp-indigent, and so torsionfree right $R$-modules are closed under submodules by our hypothesis. $(\Rightarrow)$ Let $X$ be an RDprojective module, $Y \in \mathfrak{P r}^{-1}(X)$ and $Z \subseteq Y$. Let $\nu: X \rightarrow Z$ be a homomorphism and $g: W \rightarrow Y$ an epimorphism with $W$ free. The following diagram is commutative.

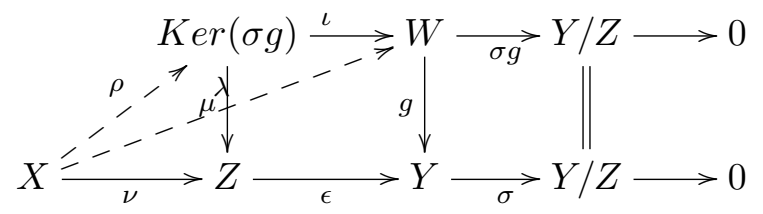

Since $Y \in \mathfrak{P r}^{-1}(X)$, we have a homomorphism $\lambda: X \rightarrow W$ such that $g \lambda=\epsilon \nu$, where $\epsilon$ is the inclusion. $R$ is torsionfree, hence $\operatorname{Ker}(\sigma g)$ is torsionfree as a submodule of the projective module $W$. By factor theorem, since $(\sigma g) \lambda=\sigma i \nu=0 \nu=0$, we have $\rho: X \rightarrow$ $\operatorname{Ker}(\sigma g)$ such that $\iota \rho=\lambda$. Then $\epsilon \nu=g \lambda=g \iota \rho=\epsilon \mu \rho$, and this implies that $\nu=\mu \rho$. By [15, Lemma 1], $X$ is $Z$-subprojective.

A module $X$ is embeded in a projective module if and only if $X$ is $E(X)$-subprojective ([13, Lemma 2.3]). We improved this result as follows:

Proposition 2.6. For any right $R$-module $Y$, the following are equivalent:

(1) $Y$ embeds in a free module.

(2) Injective right $R$-modules are in $\mathfrak{P r}^{-1}(Y)$.

(3) Every right $R$-module is embeded in a module $X$ in $\underline{\mathfrak{P r}}^{-1}(Y)$.

(4) $Y$ is embeded in a module $X$ in $\mathfrak{P r}^{-1}(Y)$. 
Proof. (1) $\Rightarrow(2)$ by [13, Lemma 2.3]. (2) $\Rightarrow$ (3) This follows by the fact that every module is embeded in an injective module. (3) $\Rightarrow(4)$ is clear. (4) $\Rightarrow(1)$ Let $\iota: Y \rightarrow X$ be an inclusion homomorphism. Consider the epimorphism $p: W \rightarrow X$ with $W$ free module. Since $X \in \mathfrak{P r}^{-1}(Y)$, there is a homomorphism $f: Y \rightarrow W$ such that $p f=\iota$. However, $\iota$ is a monomorphism, hence $f$ is also a monomorphism. This proves our claim.

A sequence $0 \rightarrow A_{1} \rightarrow A_{2} \rightarrow A_{3} \rightarrow 0$ of left modules is neat (resp., s-pure) exact if for each simple module ${ }_{R} S$ (resp., $J_{R}$ ), the sequence $\operatorname{Hom}\left(S, A_{2}\right) \rightarrow \operatorname{Hom}\left(S, A_{3}\right) \rightarrow 0$ (resp., $0 \rightarrow J \otimes A_{1} \rightarrow J \otimes A_{2}$ ) is exact. The definitions of neat submodule and s-pure submodule coincide with closed submodule over commutative hereditary noetherian rings (see [21]). A complete characterization of commutative rings for which each simple module is RD-injective is given in [10]. The following proposition follows by [24, Lemma 2.1] and [10, Theorem IV.1].

Proposition 2.7. The following statements are equivalent.

(1) RD-exact sequences of left modules are neat.

(2) Simple left modules are RD-projective.

(3) Each simple left modules is both f.p. (or pure-projective) and RD-flat.

(4) RD-exact sequences of right $R$-modules are s-pure and simple left modules are f.p. (or pure-projective).

When $R$ is commutative, these conditions are equivalent to that each simple module is both f.p. and RD-injective.

Proof. (1) $\Rightarrow(2)$ and (3) $\Rightarrow(4)$ are clear. (2) $\Rightarrow(3)$ follows by [31, Corollary 1]. (4) $\Rightarrow$ (1) By [24, Lemma 2.1(2)], every simple left $R$-module is RD-projective, implying that every RD-exact sequences of left module is neat. The commutative case follows by [10, Theorem IV.1].

We call $R$ a left SRDP ring if it satisfies the equivalent conditions of Proposition 2.7. Similarly one defines right SRDP rings. A ring in which all right ideals are principal is called a principal right ideal ring, or right PIR. PIR rings are obviously SRDP. Less obvious example of SRDP ring is commutative hereditary noetherian ring (see [7, Theorem $2.14])$.

Proposition 2.8. Let $\Delta$ be a set of all representatives of singular simple modules over commutative hereditary noetherian ring $R$. Then $\oplus_{S \in \Delta} S$ is both $R D$-projective and srdpindigent module.

Proof. Over commutative hereditary noetherian ring, simple modules are RD-projective by [7, Theorem 2.14 ]. Since RD-projective modules closed under direct sums, the module $\oplus_{S \in \Delta} S$ is an RD-projective. Now, the module $\oplus_{S \in \Delta} S$ is srdp-indigent by [14, Proposition $5.2]$.

\section{Every non-projective RD-projective module is rdp-indigent}

In this section, we studied rings whose (singular simple) RD-projective modules are rdp-indigent. As in [3] and [18], PP rings have an important role in our studies. A left $\mathrm{PP}$ ring is a ring whose all principal left ideals are projective. Note that every right (or left ) PP ring is a torsionfree ring, and the converse is true when the right annihilators of elements are f.g. [11, Theorem 4.5]. For convenience,

(S) stands for the property that non-projective simple right $R$-modules are rdp-indigent.

Lemma 3.1. If $R$ is a right SRDP ring satisfying property (S), then it is either left divisible or left $P P$. 
Proof. Consider two nonisomorphic singular simple modules $U_{R}$ and $V_{R}$. Clearly, $U$ is $V$-subprojective, and thus $V$ would be torsionfree. But $V$ is RD-projective, and so it is projective by [24, Corollary 2.5]. This contradicts with singularity of $V$. Therefore, $U$ is unique. Then either $\operatorname{Hom}_{R}(U, R)=0$ or $\operatorname{Hom}_{R}(U, R) \neq 0$. If $\operatorname{Hom}_{R}(U, R) \neq 0, U$ embeds in $R$, and by Proposition 2.6, every injective module is torsionfree. In particular, the injective module ${ }_{R} R^{+}$is torsionfree, and hence $R^{++}$is divisible by standart adjoint isomorphism. Then $R$ is a left divisible since it is (RD-)purely embeds in $R^{++}$by [16, Proposition 5.3.9]. If $\operatorname{Hom}_{R}(U, R)=0$, then $\operatorname{Hom}(U, G)=0$ for each ideal $G$ of $R$. Then, $U$ is $G$-subprojective, and so $G$ is torsionfree, implying that $R$ is torsionfree. Now, let $\left\{N_{i}\right\}_{i \in \Phi}$ be a class of torsionfree right $R$-modules. Since $U$ is $\operatorname{rdp}$-indigent and $\operatorname{Hom}(U, R)=0$, a module $H$ is torsionfree if and only if $\operatorname{Hom}_{R}(U, H)=0$. Set $H:=\prod_{i \in \Phi} N_{i}$. Now, $H_{R o m}\left(U, \prod_{i \in \Phi} N_{i}\right) \cong \oplus_{i \in \Phi} H o m_{R}\left(U, N_{i}\right)=0$, and so $H$ is torsionfree. By [11, Theorem 4.6], $R$ is a left $\mathrm{PP}$ ring.

Note that rings with property $(\mathrm{S})$ must have a unique singular simple right $R$-module. In the sequel, this simple module will be denoted by $U_{R}$ throughout paper.

Recall that a module is called coatomic provided that every submodule is contained in a maximal submodule. Semisimple modules and f.g. modules are well-known examples of coatomic modules.

Proposition 3.2. If $R$ is a right SRDP right nonsingular ring but not left divisible, then the following are equivalent:

(1) For all singular coatomic module $Z_{R}, \mathfrak{P r}^{-1}(Z) \subseteq \mathcal{T F}_{R}$.

(2) $R$ satisfies $(S)$.

Proof. (1) $\Rightarrow(2)$ is clear. (2) $\Rightarrow$ (1) It is enough to show that if a singular coatomic right module $Z$ is subprojective to a module $H$, then $H$ is torsionfree. By Lemma 3.1, $R$ is torsionfree ring. Let $Z$ be a singular coatomic right module. Since $Z$ is coatomic and singular, it has a maximal submodule $W$ such that $Z / W$ is singular and simple. By uniqueness of $U_{R}, Z / W \cong U_{R}$, implying that $\operatorname{Hom}_{R}(Z, U) \neq 0$. Now let $H$ be a module in $\mathfrak{P r}^{-1}(Z)$. Since $U$ is simple, either $\operatorname{Hom}_{R}(U, H)=0$ or $\operatorname{Hom}_{R}(U, H) \neq 0$. The later case is not possible, since if $\operatorname{Hom}_{R}(U, H) \neq 0$, then $U$ is isomorphic to a submodule of $H$. Then, by Proposition 2.5, $Z$ is $U$-subprojective, and this would imply that $H_{o} m_{R}(Z, U)=0$ by nonsingularity of $R_{R}$, a contradiction. If $\operatorname{Hom}_{R}(U, H)=0$, then $U$ is $H$-subprojective. Thus, $H$ is torsionfree, since $U$ is rdp-indigent. Therefore, $Z$ is an rdp-indigent.

Lemma 3.3. If $R$ is a right $S R D P$ but not left divisible ring, then $R$ is satisfying property $(S)$ if and only if all non-projective $R D$-projective right $R$-modules of finite length are rdp-indigent.

Proof. The sufficiency is clear. For the necessity, let $X_{R}$ be a non-projective RD-projective module of finite length. Let us point out that $R$ is torsionfree ring by Lemma 3.1. Let $Y \in \mathfrak{P r}^{-1}(X)$. If $\operatorname{Hom}_{R}(U, Y)=0$, then $Y$ is clearly torsionfree by our hypothesis. If $\operatorname{Hom}_{R}(U, Y) \neq 0$, noting that $U$ is simple, then $X$ is $U$-subprojective by Proposition 2.5. Then, $X$ is subprojective to each simple right $R$-module. $X$ has a composition series $0=U_{0} \subseteq U_{1} \subseteq U_{2} \subseteq \ldots \subseteq U_{t}=X$ with $U_{i} / U_{i-1}$ simple for each $i \in\{0,1, \ldots, t\}$. Consider the sequence $0 \rightarrow U_{1} \rightarrow U_{2} \rightarrow U_{2} / U_{1} \rightarrow 0$. Since $U_{1}, U_{2} / U_{1} \in \mathfrak{P r}^{-1}(X), U_{2} \in \mathfrak{P r}^{-1}(X)$. Similarly one can show that $U_{i} \in \mathfrak{P r}^{-1}(X)$ for each $i$. In particular, $X$ is subprojective to $U_{t}=X$, implying that $X$ is projective. Thus, the case $\operatorname{Hom}_{R}(U, Y) \neq 0$ is not possible, and $X$ is rdp-indigent.

Note that by [10, Theorem IV.1] and [24, Lemma 2.1(2)], any pure-projective module of finite length is RD-projective over a commutative SRDP ring. 
Corollary 3.4. If $R$ is commutative SRDP but not divisible ring, then $R$ is satisfying property $(S)$ if and only if each non-projective pure-projective module of finite length is rdp-indigent.

A ring $R$ is said to be right GV-ring if all singular simple right $R$-modules are injective.

Lemma 3.5. If $R$ is a right SRDP right $G V$ but not left divisible ring, then $R$ is satisfying property $(S)$ if and only if every non-projective $R D$-projective right $R$-module is rdp-indigent.

Proof. The sufficiency is clear. For the necessity, let $W_{R}$ be a non-projective RDprojective module. Let us point out that $R$ is torsionfree ring by Lemma 3.1. Note that $\operatorname{Hom}_{R}(U, W) \neq 0$, otherwise $W$ is torsionfree, and thus making $W$ projective. Now let $Y \in \mathfrak{P r}^{-1}(W)$. Either $\operatorname{Hom}_{R}(U, Y)=0$ or $\operatorname{Hom}_{R}(U, Y) \neq 0$. In the former case, $Y$ is torsionfree. The latter case is not possible. Because if $\operatorname{Hom}_{R}(U, Y) \neq 0$, then, noting that $U$ is simple, $W$ is $U$-subprojective by Proposition 2.5. Now consider the following diagram:

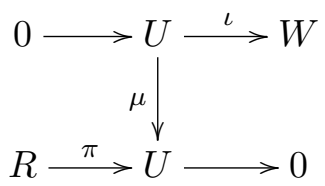

Since $R$ is a right GV-ring, $U$ is injective, and there exists a homomorphism $\varphi: W \rightarrow U$ such that $\varphi \iota=\mu$. Since $U \in \mathfrak{P r}^{-1}(W)$, there exists a homomorphism $\lambda: W \rightarrow R$ such that $\pi \lambda=\varphi$. However, the homomorphism $\lambda \iota: U \rightarrow R$ must be zero since $R$ is torsionfree ring and $U$ is singular RD-projective module, implying that $0=\pi \lambda \iota=\varphi \iota=\mu$, a contradiction. Thus, the case $\operatorname{Hom}_{R}(U, Y) \neq 0$ is not possible, and $W$ is rdp-indigent.

Proposition 3.6. If $R$ is a right SRDP but not left divisible ring, then $R$ is satisfying property $(S)$ if and only if the following hold:

(a) A module $M_{R}$ is torsionfree if and only if $\operatorname{Soc}\left(M_{R}\right)$ is projective.

(b) $R$ is a left $P P$ ring with a unique singular simple module $U_{R}$.

Proof. If $R$ satisfies (S), (b) follows by Lemma 3.1. Since $R$ is a right SRDP and $U_{R}$ is rdp-indigent, $\mathfrak{P r}^{-1}(U)=\mathcal{T F}_{R}$. It is clear that $U_{R}$ is subprojective to a module $N_{R}$ if $\operatorname{Soc}(N)$ is projective. Let $X_{R}$ be a torsionfree module and assume that $\operatorname{Soc}\left(X_{R}\right)$ is not projective. Then, by the uniqueness of $U, U \cong T$, where $T$ is a submodule of $X$. Since $R$ is torsionfree ring and $X$ is torsionfree, $U$ would be torsionfree. However, $U$ is RD-projective, hence it is projective. This contradicts with singularity of $U$. To converse, assuming (a) and (b) and let $H \in \mathfrak{P r}^{-1}(U)$. It will be enough to show that $\operatorname{Soc}(H)$ is projective. Assume contrary that $S \overline{o c}(H)$ is not projective. Then there is a submodule $N$ of $H$ such that, by uniqueness of $U, N \cong U$. R is torsionfree since it is left PP ring. Since $H \in \mathfrak{P r}^{-1}(U), U$ is $U$-subprojective by Proposition 2.5, hence $U$ would be projective. Again this contradicts with singularity of $U$.

Lemma 3.7. If $R$ is a right $S R D P$ but not left $P P$ ring with propert $(S)$, then the following hold:
a) Injective right $R$-modules are torsionfree.
b) $R$ is a left divisible.
c) Every $R D$-projective right $R$-module is embeded in a projective module.
d) $R$ is a right Kasch ring.

Proof. By Lemma 3.1, $R R$ is divisible. $\operatorname{Hom}_{R}\left(U_{R}, R\right) \neq 0$, otherwise $R$ would be a left PP ring as in the proof of Lemma 3.1. Therefore, $R$ is a right Kasch ring. Then, by Proposition 2.6, $U$ is $I_{R}$-subprojective for each injective module $I_{R}$. Therefore, noting that 
$\mathfrak{P r}^{-1}(U)=\mathcal{T F}$, injective right $R$-modules are torsionfree. In order to show that every RDprojective right $R$-module is embeded in a free module, let $H$ be an RD-projective module. Consider the inclusion $\iota: H \rightarrow E(H)$ and an epimorphism $p: W \rightarrow E(H)$ with $W$ free module. Since $H$ is RD-projective and $E(H)$ is torsionfree, there is a homomorphism $\psi: H \rightarrow W$ such that $\iota=p \psi$. Thus, $\psi$ is a monomorphism, as desired.

A module $W$ is called i-test module if $\operatorname{Ext}_{R}^{1}(W, T) \neq 0$ for all non-injective module $T$. A nonsemisimple ring is right n-saturated if its non-projective f.g. right $R$-modules are i-test ([30]).

Theorem 3.8. Let $R$ be a right $S R D P$ ring without an infinite family of orthogonal idempotents. If $R$ is not right Kasch and it satisfies (S), then the following hold:

a) Non-torsionfree right $R$-modules are $i$-test.

b) Divisible (m-injective) right $R$-modules are injective.

c) The classes of torsionfree and nonsingular right $R$-modules coincide.

d) $R$ is a right hereditary right noetherian right $C$-ring.

Proof. By Lemma 3.1 and Lemma 3.7, $R$ is a left PP ring. Let us point out that, by our hypothesis, $\mathfrak{P r}^{-1}\left(U_{R}\right)=\mathcal{T F}$ and, by singularity of $U, U$ is subprojective to $M$ where $M$ is nonsingular. $R$ is a right PP ring by [8, Lemma 8.4], and hence, by [3, Corollary 3.4], all torsionfree right $R$-modules are nonsingular. Then, the classes of torsionfree and nonsingular right $R$-modules coincide. Let $W_{R}$ be an m-injective module. There is a sequence $0 \rightarrow W \rightarrow E(W) \rightarrow E(W) / W \rightarrow 0$. $\operatorname{Soc}(E(W) / W)$ is projective, otherwise $E(W) / W$ has a singular simple submodule, say $C$. Then $E(W)$ has an essential submodule $L$ such that $L / W \cong C$. Since $U$ is unique, $U \cong C$, hence $\operatorname{Ext}(C, W)=0$. Then $W$ is a direct summand in $L$, and this contradicts with essentiality of $W$ in $L$. Therefore, $\operatorname{Soc}(E(X) / X)$ is projective and, by Proposition 3.6, $E(X) / X$ is nonsingular. $M$ is closed in $E(M)$, thus it is injective. This is equivalent to $R$ is a right $C$-ring. Moreover, since $R$ is also right SRDP, every divisible (and fp-injective) right $R$-module is injective, implying right Noetherianity of $R$. Since $R$ is a right PP, injective modules are closed under quotients by [24, Corollary 2.13], and this would imply that $R$ is also right hereditary. If a module $T_{R}$ is non-torsionfree, then either $\operatorname{Hom}_{R}(U, T)=0$ or $\operatorname{Hom}_{R}(U, T) \neq 0$. The former is impossible, since $U$ is $T$-subprojective and $U$ is rdp-indigent. Then $\operatorname{Hom}_{R}(U, T) \neq 0$. Since $U$ is simple, w.l.o.g. we may take $U$ as a submodule of $T$. Then, since $R$ is hereditary and $U$ is i-test, $T$ is i-test by [14, Proposition 4.3].

For easy reference, we define the following for $R$ :

(Q) Every non-projective simple right $R$-module is srdp-indigent.

Corollary 3.9. If $R$ is a right SRDP ring with property (Q), then it is right IF ring or its weak dimension is $\leq 1$.

Proof. By our hypothesis, $\mathcal{T F}_{R}=\mathcal{F}_{R}$. By Lemma 3.1, $R$ is either a left fp-injective ring or a left PP ring. In the former case, injective right $R$-modules are flat, i.e. $R$ is a right IF by Lemma 3.7. In the latter case, since $\mathcal{T F}_{R}=\mathcal{F}_{R}$, all right ideals of $R$ are flat, i.e. the weak dimension of $\mathrm{R}$ is $\leq 1$.

An $R$-module $V$ is extending (or CS) provided each of its closed submodules is direct summand of $V$. Similar to $\Sigma$-injective modules, $K$ is called a (finitely) $\Sigma$-CS module if all direct sum of (finitely many) copies of $K$ is CS (see [12]).

Lemma 3.10. If $R$ is a right $S R D P$ but not IF ring without an infinite family of orthogonal idempotents, then $R$ satisfies ( $Q$ ) if and only if the following cases occur:

(i) $R$ is a right hereditary right noetherian right $C$-ring with unique simple singular module $V_{R}$.

(ii) $R$ is a right finitely $\Sigma$-CS ring. 
Proof. $(\Rightarrow)$ (i) follows by Theorem 3.8. Let $W$ be a f.g projective module and $H$ a closed submodule of $W$. Note that closed submodules are neat by $[9,10.10]$. Then $V$ is $\frac{W}{H}$-subprojective. Since $V$ is srdp-indigent, $\frac{W}{H}$ is flat module. Let us point out that $\frac{W}{H}$ is finitely presented since $R$ is a right noetherian. Thus $\frac{W}{H}$ is a projective module, implying that $H$ is a direct summand of $W$. Therefore, $R_{R}$ is finitely $\Sigma$-CS. $(\Leftarrow)$ Let $X \in \mathfrak{P r}^{-1}(V)$. We claim that $X$ is a flat module. Since $R_{R}$ is nonsingular and $V$ is singular, $\operatorname{Hom}_{R}(V, X)=0$. Assume contrary that $X$ is not flat, then, by [28, Corollary 3.49], $X$ has a f.g. non-flat submodule, say $A$. $\operatorname{Since} \operatorname{Hom}(V, X)=0, \operatorname{Hom}(V, A)=0$. Now, consider the singular submodule $Z(A)$ of $A$. However, $R$ is a right $C$-ring and $V$ is unique. The singular submodule $Z(A)$ of $A$ has submodule $K$ such that $K \cong V$, implying $\operatorname{Hom}(V, A) \neq 0$, a contradiction. Therefore $Z(A)=0$, hence $A$ is a nonsingular. Since $R_{R}$ is finitely $\Sigma$-CS, $A$ must be projective by [12, Corollary 11.4], a contradiction. Therefore, $X$ is a flat module by [28, Corollary 3.49].

Theorem 3.11. If a ring $R$ is left Noetherian right $C$-ring and right SRDP ring with property (Q), then only one of the following cases occurs:

(i) a) Non-torsionfree right $R$-modules are $i$-test.

b) Divisible (m-injective) right $R$-modules are injective.

c) The classes of torsionfree right $R$-modules and nonsingular right $R$-modules coincide.

d) $R$ is a right hereditary right noetherian.

(ii) $R \cong R_{1} \times R_{2}$, where $R_{1}$ is a semisimple artinian and $R_{2}$ is an indecomposable $n$-saturated ring which is a matrix ring over a local QF-ring.

Proof. By Corollary 3.9, $R$ is either left fp-injective or $w D(R) \leq 1$. Since $R$ is a right $C$-ring and right SRDP ring, every divisible (fp-injective, m-injective) right $R$-module is injective, implying that $R$ is a right noetherian. In the case of $w D(R) \leq 1$, (i) follows. On the other hand, since $w D(R) \leq 1$, every right ideal of $R$ is flat. Since $R$ is a right noetherian, every right ideal of $R$ is finitely presented, implying that $R$ is a right hereditary. It remains to show (i-c) and (i-a). For (i-c), since $\operatorname{Hom}(U, N)=0$ for each nonsingular module $N$ and $U$ is srdp-indigent, all nonsingular right $R$-modules are torsionfree (and flat). Let $X_{R}$ be a torsionfree module. There is a sequence $0 \rightarrow A_{1} \rightarrow A_{2} \rightarrow X \rightarrow 0$ with $A_{2}$ free. This sequence is closed exact since $\mathfrak{P r}^{-1}(U)=\mathcal{T F}$ and $R$ is a right $C$-ring. Since $R$ is a right nonsingular, $A_{2}$ is nonsingular, and so $X$ would be nonsingular by [29, Lemma 2.3]. For (i-a), let $Y$ be a non-torsionfree right $R$-module. $\operatorname{Hom}(U, Y) \neq 0$, otherwise $U$ is $Y$-subprojective, hence $Y$ is torsionfree, a contradiction. Then, noting that $U$ is simple, $U$ is isomorphic to a submodule of $Y$. Let us point out that, $U$ is an i-test module, since $R$ is a $C$-ring. Since $R_{R}$ is hereditary and $U$ is i-test, $Y$ is i-test by [14, Proposition 4.3]. Recall that every fp-injective right $R$-module is injective if and only if $R$ is a right Noetherian ([26, Theorem 3]). If ${ }_{R} R$ is fp-injective, then $R$ is a $Q F$-ring since $R$ is a left noetherian by [22, Theorem 15.1]. By our hypothesis, $\mathfrak{P r}^{-1}(N)$ would be the class of projective right $R$-modules, i.e. $N$ is p-indigent. Now (ii) follows by [13, Theorem 3.1].

Corollary 3.12. If $R$ is a left Noetherian right PIR ring with property (Q), then only one of the following cases occurs:

(i) The following statements hold.

a) $R$ is a right hereditary right noetherian.

b) Every divisible (m-injective) right $R$-module is injective.

c) The classes of torsionfree right $R$-modules, flat right $R$-modules and nonsingular right $R$-modules coincide.

d) Every non-torsionfree right $R$-module is i-test.

(ii) $R \cong R_{1} \times R_{2}$, where $R_{1}$ is a semisimple artinian ring and $R_{2}$ is an indecomposable $n$-saturated ring which is matrix ring over a local QF-ring. 
Proof. A right PIR ring satisfying the condition (Q) is a right C-ring. By the condition $(\mathrm{Q}), \mathcal{T}_{\mathcal{F}}=\mathcal{F}_{R}$, and hence $\mathfrak{P r}^{-1}(U)=\mathcal{F}$. Let $X_{R}$ be an m-injective module, equivalently, $\operatorname{Ext}_{R}^{1}(U, X)=0$ by uniqueness of $U$. $\operatorname{Soc}(E(X) / X)$ is projective as in the proof of Theorem 3.8. Then $\operatorname{Hom}_{R}\left(U, \frac{E(X)}{X}\right)=0$. Since $\mathfrak{P r}^{-1}(U)=\mathcal{F}, \frac{E(X)}{X}$ is flat, hence $X$ is fp-injective. By right noetherianity, $X$ is injective, implying that $R$ is a right $C$-ring. Now, the claim follows by Theorem 3.11.

Theorem 3.13. If $R$ is a two-side noetherian right $S R D P$ ring satisfying property $(Q)$, then $R \cong R_{1} \times R_{2}$, where $R_{1}$ is a semisimple artinian and $R_{2}$ is an indecomposable noetherian ring satisfying only one of the following statements:

(i) $R_{2}$ is a right matrix ring over local QF-ring; or,

(ii) $R_{2}$ is a prime hereditary ring; or,

(iii) $R_{2}$ is a Morita-equivalent to a lower triangular matrix ring over a division algebra.

Proof. Following Corollary 3.9, $\mathrm{R}$ is either $w D(R) \leq 1$ or left fp-injective. Since $U$ is singular, every nonsingular module is flat by our hypothesis. By mimicking the proof of the previous corollary, it can be easily shown that m-injective right $R$-modules are fpinjective. However, $R$ is a right Noetherian, all $\mathrm{m}$-injective right $R$-modules are injective, i.e. $R$ is a right $C$-ring. If $w D(R) \leq 1$, noting that $R$ is two-sided noetherian, $R$ is two-sided hereditary. Now, since $U$ is a srdp-indigent, the classes of torsionfree right $R$-modules and flat right $R$-modules are the same. Moreover, the class of flat right $R$ modules coincides with the class of nonsingular right $R$-modules, since flat modules are nonsingular by [14, Proposition 4.4]. By [3, Theorem 5.5], $R \cong R_{1} \times R_{2}$, where $R_{1}$ is semisimple Artinian ring and $R_{2}$ satisfies either (ii) or (iii). If ${ }_{R} R$ is fp-injective, then $R$ is $\mathrm{QF}$, thus $U$ is p-indigent. Now, (i) follows by [13, Theorem 3.1].

Acknowledgment. This research was supported by TUBITAK 1002-Short Term R\&D Funding Program (Project number: 118F311). The author would like to thank the referees for their valuable comments.

\section{References}

[1] Y. Alagöz and Y. Durğun, An alternative perspective on pure-projectivity of modules, São Paulo J. Math. Sci. 14 (2), 631-650, 2020.

[2] A.N. Alahmadi, M. Alkan and S.R. López-Permouth, Poor modules: The opposite of injectivity, Glasgow Math. J. 52A, 7-17, 2010.

[3] U. Albrecht, J. Dauns and L. Fuchs, Torsion-freeness and non-singularity over right p.p.-rings, J. Algebra, 285 (1), 98-119, 2005.

[4] R. Alizade and Y. Durğun, Test modules for flatness, Rend. Semin. Mat. Univ. Padova, 137, 75-91, 2017.

[5] P. Aydoğdu and S. R. López-Permouth, An alternative perspective on injectivity of modules, J. Algebra, 338, 207-219, 2011.

[6] P. Aydoğdu and B. Saraç, On artinian rings with restricted class of injectivity domains, J. Algebra, 377, 49-65, 2013.

[7] M. Behboodi, A. Ghorbani, A. Moradzadeh-Dehkordi and S.H. Shojaee, C-pure projective modules, Comm. Algebra, 41 (12), 4559-4575, 2013.

[8] A.W. Chatters and C.R. Hajarnavis, Rings with chain conditions, Pitman, Boston, Mass.-London, 1980.

[9] J. Clark, C. Lomp, N. Vanaja and R. Wisbauer, Lifting modules, Birkhäuser Verlag, Basel, 2006.

[10] F. Couchot, RD-flatness and RD-injectivity, Comm. Algebra, 34 (10), 3675-3689, 2006.

[11] J. Dauns and L. Fuchs, Torsion-freeness for rings with zero divisor, J. Algebra Appl. 3 (3), 221-237, 2004.

[12] N.V. Dung, D.V. Huynh, P.F. Smith and R. Wisbauer, Extending modules, Longman Scientific \& Technical, 1994.

[13] Y. Durğun, Rings whose modules have maximal or minimal subprojectivity domain, J. Algebra Appl. 14 (6), 1550083, 2015. 
[14] Y. Durğun, An alternative perspective on flatness of modules, J. Algebra Appl. 15 (8) 1650145, 2016.

[15] Y. Durğun, Subprojectivity domains of pure-projective modules, J. Algebra Appl. 19 (5), 2050091, 2020.

[16] E. E. Enochs and O. M. G. Jenda Relative homological algebra, Walter de Gruyter \& Co., 2000.

[17] A. Facchini and A. Moradzadeh-Dehkordi, Rings over which every RD-projective module is a direct sums of cyclically presented modules, J. Algebra 401, 179-200, 2014.

[18] A. Hattori, A foundation of torsion theory for modules over general rings, Nagoya Math. J. 17, 147$158,1960$.

[19] C. Holston, S.R. López-Permouth and N.O. Ertaş, Rings whose modules have maximal or minimal projectivity domain, J. Pure Appl. Algebra, 216 (3), 673-678, 2012.

[20] C. Holston, S.R. López-Permouth, J. Mastromatteo and J.E. Simental-Rodriguez, An alternative perspective on projectivity of modules, Glasgow Math. J. 57 (1), 83-99, 2015.

[21] K. Honda, Realism in the theory of abelian groups. I, Comment. Math. Univ. St. Paul. 5, 37-75, 1956.

[22] T.Y. Lam, Lectures on modules and rings, Springer-Verlag, 1999.

[23] S.R. López-Permouth, J. Mastromatteo, Y. Tolooei and B. Ungor, Pure-injectivity from a different perspective, Glasg. Math. J. 60 (1), 135-151, 2018.

[24] L. Mao, Properties of RD-projective and RD-injective modules, Turkish J. Math. 35 (2), 187-205, 2011.

[25] L. Mao and N. Ding, On divisible and torsionfree modules, Comm. Algebra, 36 (2), 708-731, 2008.

[26] C. Megibben, Absolutely pure modules, Proc. Amer. Math. Soc. 26, 561-566, 1970.

[27] P. Rothmaler, Torsion-free, divisible, and Mittag-Leffler modules, Comm. Algebra 43 (8), 3342-3364, 2015.

[28] J. Rotman, An introduction to homological algebra, Academic Press, 1979.

[29] F. L. Sandomierski, Nonsingular rings, Proc. Amer. Math. Soc. 19, 225-230, 1968.

[30] J. Trlifaj, Whitehead test modules, Trans. Amer. Math. Soc. 348 (4), 1521-1554, 1996.

[31] R. B. Warfield, Purity and algebraic compactness for modules, Pacific J. Math. 28, 699-719, 1969. 